This is a self-archived version of an original article. This version may differ from the original in pagination and typographic details.

Author(s): Kujala, Tuomo; Mäkelä, Jakke

Title: Naturalistic study on the usage of smartphone applications among Finnish drivers

Year: 2018

Version: Accepted version (Final draft)

Copyright: @ 2018 Elsevier Ltd.

Rights: $C C$ BY-NC-ND 4.0

Rights url: https://creativecommons.org/licenses/by-nc-nd/4.0/

Please cite the original version:

Kujala, T., \& Mäkelä, J. (2018). Naturalistic study on the usage of smartphone applications among Finnish drivers. Accident Analysis and Prevention, 115, 53-61.

https://doi.org/10.1016/j.aap.2018.03.011 


\section{Naturalistic study on the usage of smartphone applications among Finnish 2 drivers}

3

4 Tuomo Kujala ${ }^{1 *}$ and Jakke Mäkelä ${ }^{1}$

5

$6 \quad{ }^{1}$ University of Jyväskylä, Jyväskylä, Finland

$7 \quad{ }^{*}$ Corresponding author: tuomo.kujala@jyu.fi, P.O. Box 35, 40014 University of Jyväskylä, 8 Finland

9

0

1

2

Abstract

We present results from a naturalistic study that tracked how Finnish drivers use their smartphones while driving. We monitored 30 heavy-user drivers in Finland in June-September 2016, and recorded the times when they used their phones, the application used at the time of touch, and the location and speed of the car. Touches were used as a proxy for estimating visual distraction due to visual-manual tasks. Our data set allows us to determine whether drivers use their phones differently on different road types (highway, main road, local rural road, urban road). We found that the road type has an effect on phone use but the effect is to the opposite direction than expected. The drivers produced more touches per hour on urban roads but the instances of use tend to be shorter than on the highway or on main roads. We also collected statistics on the applications that were used. By far the largest amount of distraction is caused by the WhatsApp messaging service, used by a majority of the drivers. An instance of WhatsApp use included a median of 8 touches, and had a median duration of 35 seconds. By contrast, navigation application use included a median of 3 touches and lasted for 11 seconds. The findings suggest that the Finnish smartphone heavy-users do not actively modulate their phone use based on the demands of the traffic conditions and that the greatest risk from smartphone use may be currently caused by messaging applications.

Keywords: distraction; smartphone; application; road type; attentional demand; behavioural adaptation 


\section{1. Introduction}

0 Driver distraction by mobile phone use has been associated with increased safety-critical incident risk in 1 traffic (e.g., [7][15]). Although it is well known that many drivers use their smartphones to do various 2 tasks while driving (e.g., [7][15]), little to nothing is known about the actual applications they use or the 3 exact traffic conditions in which they use those applications. This information is important for determining

4 the actual risk level caused by the distraction. A given application, such as texting, may cause a very 5 serious risk of accident on a busy and congested urban road. The risk may be considerably smaller when 6 driving along a straight and nearly empty highway.

In this paper, we present findings from naturalistic driving data that enabled us to determine what applications drivers use and whether they use applications differently in different driving scenarios. Ideally, we would be able to compare what applications are used on highways, major roads, minor roads, and

0 urban traffic. The greatest limitation is that no data on the congestion or real-time traffic density is 1 available. However, the road type [8] and the associated photo material recorded during the phone use 2 provide us at least a rough indication of the visual demands of the driving scenario.

Although there is no exact way to determine the visual-manual distraction caused by any given application, we used the number of touches on the smartphone as a proxy. A touch on a touch screen is

5 almost always accompanied by a glance on the touch screen due to the limited haptic feedback of the 6 device [2][14].

Among others, Victor et al. [15] have suggested that a series of glances away from the road in a 8 short period of time may lead to safety-critical uncertainty of the task-relevant road events, even if the 9 glances off road were brief. Therefore, an application that requires a large number of touches in a short 0 time period can be considered to cause high cognitive demand on the driver. On the other hand, an 1 instance of use, of which duration is long, means that the driver is distracted for a longer time period. 2 Longer in-car tasks have been associated with increased probability of increased individual glance 3 durations off road [10][11]. 
There is evidence from naturalistic driving (e.g. [16]) as well as from on-road (e.g. [17]) and

5 simulator studies (e.g. [12]) suggesting that drivers adapt their off-road glancing behaviours according to

6 the dynamic demands of the driving task. Drivers tend to decrease their off-road glance durations and the

7 number of off-road glances when the demands of the driving task increase. The naturalistic field study of

8 Metz et al. [13] (maneuvering, German drivers) and the video-clip based study of Hancox et al. [4] also

9 suggest that drivers tend to attend to distracting activities in a situationally aware manner.

0 Based on the previous research findings, our preliminary hypothesis was that at least experienced

1 drivers should have developed a sense of acceptable risk levels, and would be able to adapt their

2 smartphone usage to the demands of the given driving conditions. We would thus expect to see differences

3 in smartphone usage between road types. In particular, we hypothesized that there would be less touches

4 on the phone and the applications would be used less often in high-demand driving scenarios. It was our

5 expectation that urban roads would present the need for most vigilance due to intersections, traffic lights

6 and other traffic (including cars, bicycles and pedestrians), and hence we would see less phone use in

7 urban conditions than on highway or main roads, even though the highways and main roads have higher

8 nominal speed limits. In order to analyse if different levels of distraction can be associated with different

9 smartphone applications, we studied the most used applications on the road as well as the number of

0 touches and the durations of application use instances. 


\section{2. Materials and Methods}

$2 \quad 2.1$ Hardware and Software

3 The results presented here are a subset of a larger experiment that studied the effects of distraction

4 warnings on smartphone use while driving. The presented data are solely from the control part of the 5 experiments, when phone usage data was simply collected without any interventions. The control phase 6 was finished by September 2016, but the experimental phase continued until December 2016, ending to a 7 web questionnaire (self-reported car and phone data presented here).

The data were collected using custom software developed by Ficonic Solutions Ltd, located in

9 Jyväskylä, Finland. The software consisted of two parts: a "Watcher" application running on Samsung

0 XCover 3 smartphones that were installed in the volunteers' cars dashboards with a double-suction cup

1 windshield car holder (see Figure 1), and a small "Observer" application that was installed on all Android

2 phones and other Android devices which the drivers reported to use while driving. The Watcher

3 application created a Wi-Fi hotspot, onto which the Observer phones connected when within range.

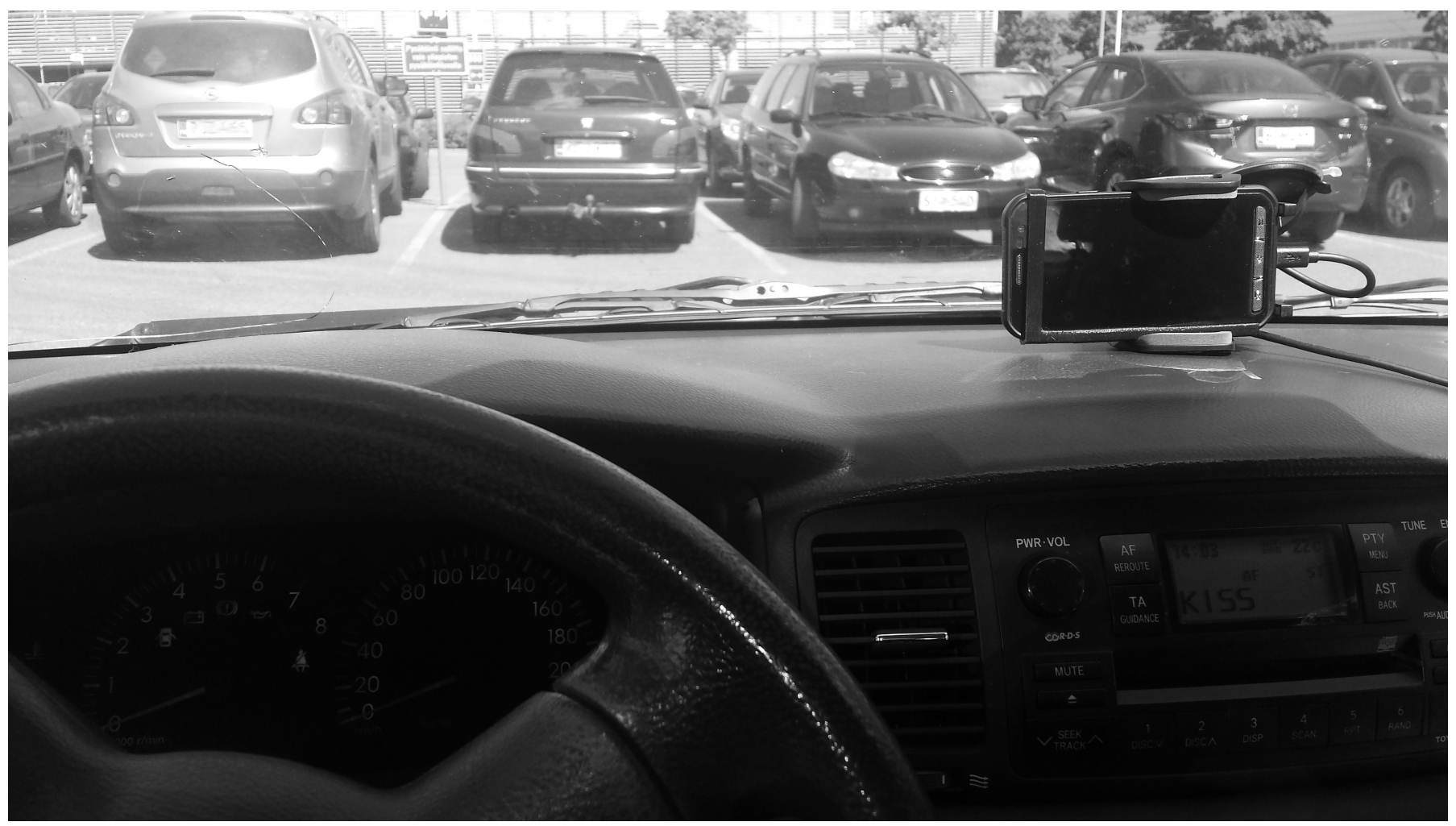

Fig. 1. The position of the dashboard smartphone with the Watcher application in a participant's car. 
The Watcher phones had a continuous cellular network connection to allow enhanced GPS

7 positioning. Data were uploaded to the remote server via $3 \mathrm{G}$ or $4 \mathrm{G}$ connection, depending on the

8 connectivity at the area, whenever the Watcher application was on standby. The Watcher application

9 recorded the GPS position at one-second intervals whenever the car was in motion. The in-built power-

0 savings system in the Android version meant that whenever the car was stationary, no GPS positions were

1 recorded.

2 In order to enable the location-based warnings in the second phase of the experiment, the Watcher 3 application constantly mapped the position of the car against the Finnish national Digiroad map data set

4 (http://www.liikennevirasto.fi/web/en/open-data/digiroad), and determined the road on which the car was

5 for any given GPS fix. The application also collected acceleration data on three axles but this data is not 6 analyzed in this paper.

The Observer background application in the drivers' Android devices worked by creating a 8 transparent layer over the other applications. A touch on the phone was thus recorded by the Observer 9 application. A flag about every touch was immediately sent over the Wi-Fi network to the Watcher phone, 0 including information about the Android front application (FrontApp) that was running at the moment. 1 The Watcher application took a photo with the back camera of the Android phone by each touch on the 2 driver's phone. The camera was positioned and secured to the windshield holder and dashboard in a way 3 that the camera had clear visibility to the road environment in front of the vehicle (see Figure 1).

The car models used by the participants during the study are listed in Table 1 and their Android 5 devices with the Observer application in Table 2 (based on self-reporting). One participant could have up 6 to three cars and three Android devices in use during the research. If the car was changed in the middle of 7 the study, the research equipment was moved to the new car. Most of the participants' cars were equipped 8 with manual transmission $(29 / 40,72.5 \%)$. 
Table 1 Participants' car models in the study.

\begin{tabular}{|c|c|c|c|}
\hline Make & Model & Year (where available) & Transmission \\
\hline Audi & A3 & NA & manual \\
\hline Audi & A4 & NA & manual \\
\hline Audi & A4 & NA & manual \\
\hline Audi & A5 & NA & automatic \\
\hline Audi & A6 & 2004 & automatic \\
\hline BMW & 518 & 2015 & automatic \\
\hline BMW & 320 & 2013 & automatic \\
\hline Chevrolet & Suburban & 1996 & automatic \\
\hline Citroen & C5 & 2008 & automatic \\
\hline Ford & Fiesta & NA & manual \\
\hline Ford & Focus & NA & manual \\
\hline Ford & Focus & 2000 & manual \\
\hline Ford & Ranger & 2016 & automatic \\
\hline Nissan & Qashqai & 2012 & manual \\
\hline Nissan & Qashqai & 2015 & manual \\
\hline Nissan & Primera & 1998 & manual \\
\hline Opel & Astra & 2004 & manual \\
\hline Peugeot & $206 \mathrm{SW}$ & 2004 & manual \\
\hline Peugeot & 308 & 2008 & manual \\
\hline Seat & Altea & 2005 & automatic \\
\hline Skoda & Octavia & 2006 & manual \\
\hline Skoda & Octavia & 2012 & automatic \\
\hline Skoda & Octavia SW & 2015 & manual \\
\hline Skoda & Rapid & 2014 & manual \\
\hline Smart & ForFour & 2006 & manual \\
\hline Subaru & Forester & 2010 & manual \\
\hline Toyota & Avensis & 1998 & manual \\
\hline Toyota & Corolla & NA & manual \\
\hline Toyota & Corolla & NA & manual \\
\hline Volkswagen & Caddy Maxi & NA & manual \\
\hline Volkswagen & Golf & 2000 & manual \\
\hline Volkswagen & Golf & 2000 & manual \\
\hline Volkswagen & Golf SW & 2003 & manual \\
\hline Volkswagen & Passat & 1998 & manual \\
\hline Volkswagen & Passat & 2000 & manual \\
\hline Volkswagen & Polo & NA & manual \\
\hline Volkswagen & Transporter & 1996 & manual \\
\hline Volvo & S80 & 1999 & manual \\
\hline Volvo & V70 & 2002 & manual \\
\hline Volvo & V70 & 2012 & automatic \\
\hline
\end{tabular}


Table 2 Participants' Android devices in the study.

\begin{tabular}{lccc}
\hline Make & Model & Count \\
\cline { 1 - 1 } \cline { 3 - 3 } CAT & B15Q & 1 \\
HTC & Desire & 1 \\
Huawei & Honor Holly & 1 \\
Huawei & Honor 7 & 3 \\
Huawei & Honor 8 & 1 \\
Huawei & Nexus 6P & 1 \\
LG & GFlex & 1 \\
LG & Nexus 5x & 2 \\
Samsung & Galaxy S4 & 4 \\
Samsung & Galaxy S5 & 3 \\
Samsung & Galaxy S5 Mini & 1 \\
Samsung & Galaxy S6 Edge & 2 \\
Samsung & Galaxy S7 & 1 \\
Samsung & Galaxy XCover 2 & 2 \\
Samsung & Galaxy J5 & 1 \\
Samsung & Galaxy Alpha & 1 \\
Sony & Xperia Z2 & 1 \\
Sony & Xperia Z3 & 1 \\
Sony & Xperia Z3+ & 3 \\
Sony & Xperia Z5 & 1 \\
Sony & Xperia X Performance & 1 \\
\hline
\end{tabular}

$6 \quad 2.2 \quad$ Participants

7 The number of volunteers recruited in the study via convenience sampling was initially 31 , starting in June 8 2016. One participant dropped out of the study before sufficient control data could be collected. The total 9 number of drivers in this study is therefore $N=30(22 \mathrm{M}, 8 \mathrm{~F}$; median age 37 , mean age $39, S D$ 12.2, range

0 18-64). Participants were recruited via ads in newspapers and social media. The recruitment ad required 1 volunteers to drive "a lot" and to "regularly" use their smartphones while driving, but no quantitative 2 criteria were given. Well over 200 applications were eventually received. The final participants were 3 selected based on multiple criteria, including how much they reported themselves to drive per year. Those 4 drivers were favored who reported driving in both urban and rural areas. An attempt was made to choose 5 as many women as possible, and to achieve a wide spread in ages. The participants got to keep the 6 Android smartphone, the car charger and the car holder as a reward for participation after the study. All 7 the participants signed a written informed consent document prior to participation and they were allowed 
8 to withdraw from the study at any point. The University of Jyväskylä Ethical Committee was enquired for

9 the need to have an ethical review for the study and the study was approved without a formal review.

0 2.3 Data Collection and Analysis

1 The initially planned number of days for the drivers to spend in the control stage was 63 days. However,

2 this was increased to 84 days (12 weeks) for the great majority of the drivers. In practice, there was a large

3 variation in the number of days that drivers actually drove during the control period, enhanced by technical

4 problems, which caused periods of data blackout. The mean number of days was 36.7 (SD 18.3), with

5 median of 39 and range from 7 to 68.

The data used in this paper are only for cases where the car was traveling with a speed of at least 2 $\mathrm{m} / \mathrm{s}(7.2 \mathrm{kmh})$. This was the lower speed limit that could be reliably detected by the GPS. Data were collected separately in cases where the car was stationary, but this data is not included in this paper. In

9 general, there was no significant difference between the number of touches while driving or while

0 stationary $(p=.870)$. The number of hours spent driving varied from 7 to 167 hours, with a median of 50

1 hours (mean 56 hours). The total number of touches recorded per driver while driving also varied 2 enormously. The range was 132 to 22,337 , with three drivers recording over 10,000 touches during the 3 control period. The median for touches was 1,538.

In order to analyze the reliability of the road classification system used in the analyses, we analyzed

5 the vehicle speeds at touch on the different road types and compared these to the roads' nominal speed

6 limits. In addition, for analyzing if the traffic densities differed by road type during the phone use, we

7 made an analysis of traffic densities in the photos taken by each touch on the drivers' phones. A random

8 sample of 310 photos collected during the control stage in between June and September 2016 was

9 manually scored by a research assistant for the number of vehicles and light traffic (pedestrians, cyclists,

0 small motorized vehicles) visible in the photo. Only those road users visible in the photos who had an

1 access to the road the participant was driving, were included in the data (e.g., on-coming cars which were

2 on an adjacent lane with a fence are excluded). Only photos with a clear view of the road environment 
3 ahead and taken in situations where the road ahead continues forward were included (i.e., no T-

4 intersections or the end of a road). Photos taken with speed below $2 \mathrm{~m} / \mathrm{s}$ were excluded from the analysis.

5 At least one photo from each different Watcher device was included in the sample.

6 The distributions of the data were mostly non-Gaussian and therefore, nonparametric tests were

7 utilized for testing the hypotheses. Where applicable, Cohen's d is reported as a measure of effect size. R

8 (version 3.3.3) and IBM SPSS Statistics (version 22) were used as tools for the statistical analyses.

$9 \quad 2.4 \quad$ Known Limitations

0 There are some caveats to the touch data. Due to technical properties of the Android operating system, the

1 front application cannot always be identified accurately. Also, touches to some applications with a

2 "floating user interface" (such as Facebook Messenger) are not captured at all as the user interface was

3 drawn over the Observer application's transparent layer. Touches related to the phone keylock are missed

4 completely. The numbers presented here are therefore lower limits. However, we estimate that the error is

5 small (0-6 touches per instance of use, that is, $19 \%$ of touches on average, if assuming the phone keylock

6 was activated between each instance of use).

Identifying an application use instance is not trivial with the data set. The Android operating system's FrontApp code returns only one application name, which sometimes can be tied to a specific

9 application but sometimes cannot. Different FrontApp names may be returned at different parts of the

0 application instances, while some FrontApp names may be related to a large number of applications.

1 Depending on the model and Android version of the driver's phone, the FrontApp might not be identified

2 at all (returning a blank, or NA). For some unidentified reason, Android hides the SMS text messages

3 behind an unknown code and these could not be identified. The ratio of unidentifiable FrontApps ranged 4 from about $3 \%$ to almost $30 \%$ for different drivers. Phone call data were collected but due to technical 5 unreliabilities in recognizing the start and end of a call the data is not reported here. 


\section{3. Results}

\section{$8 \quad 3.1 \quad$ Touches per Hour}

9 The mean touches per hour for each driver can be calculated by calculating the number of touches where

0 the speed is at least $2 \mathrm{~m} / \mathrm{s}$, and dividing by the number of GPS locations recorded for the driver with speed

1 at least $2 \mathrm{~m} / \mathrm{s}$. Since GPS locations are recorded once a second, this ratio can be multiplied by 3600 to give

2 touches per hour. The distribution is highly skewed (Figure 2), with two drivers averaging over 400

3 touches per hour. The median number of touches is 41 , meaning less than one touch per minute. The 15 th 4 and 85 th percentiles are 13 and 142 touches, respectively, and the range is 8 to 481 .

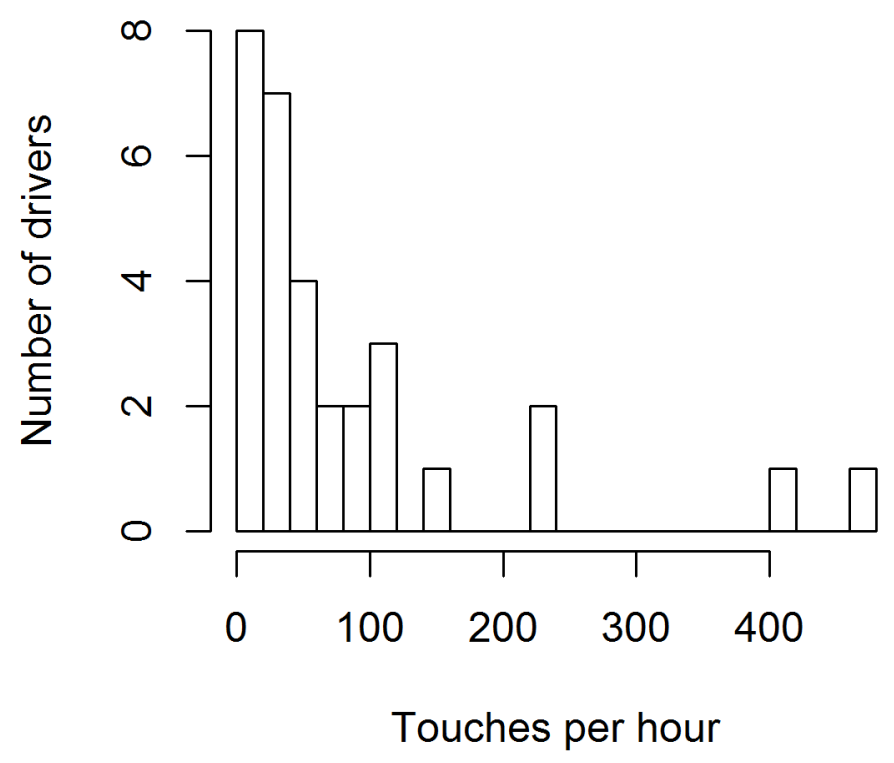

Fig. 2. Touches per hour for the 30 drivers. 


\section{$8 \quad 3.2 \quad$ Road Types and Vehicle Speed at Touch}

9 Four classes of road were a priori identified from the data based on the Digiroad classification system:

1. Highway (default $120 \mathrm{kmh}$, sometimes $100 \mathrm{kmh}$ ). No opposing traffic.

2. Main rural road (default $80 \mathrm{kmh}$, sometimes $60 \mathrm{kmh}$ [at some crossroads] or $100 \mathrm{kmh}$ ). By default the driver has right-of-way.

3. Local rural road (speed limit varies, default either $80 \mathrm{kmh}$ or $50 \mathrm{kmh}$ ). Driver may or may not have right-of-way.

In order to analyze the reliability of our classification system, we analyzed the vehicle speeds at

8 touch on the different road types. The mean speed at touch depends on the road type, as seen in Figure 3.

9 The mean speed $(S D)$ for highway touches is $101(11) \mathrm{kmh}$, main road $80(6)$, local 60 (11), and urban 35

0 (12) $\mathrm{kmh}$. These are very close to the nominal maximum speeds allowed on these road types. The finding

1 gives support for our road classification system.

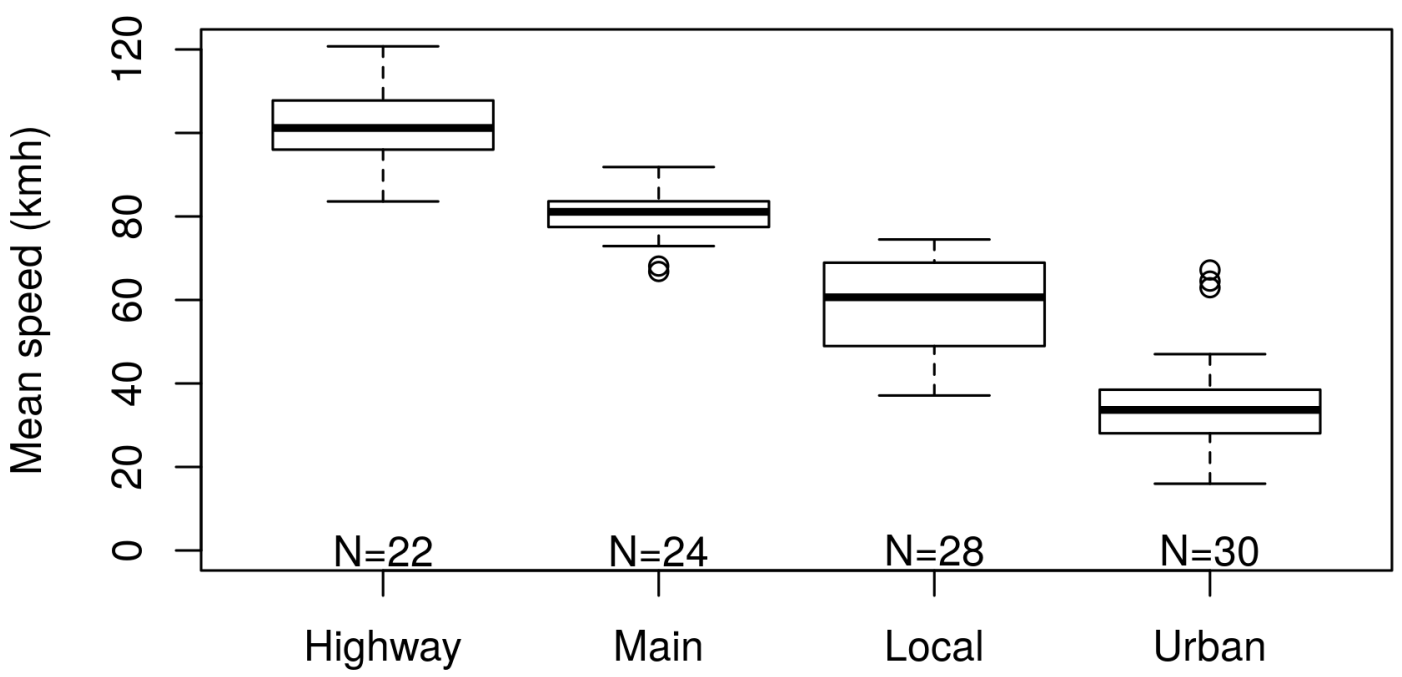

Fig. 3. Mean speed at touch by road type (kmh). 


\section{$5 \quad 3.3 \quad$ Traffic Density in Photos by Road Type}

6 In order to analyse if the traffic densities varied by road type during the phone use, we analysed a sample

7 of the photos taken from the road environment ahead of the vehicle by each touch on a driver's phone. The

8 sample size for the road type Main was too low (4 photos) for meaningful analysis, so we compared only

9 the differences in the number of vehicles and the amount of light traffic (including pedestrians, cyclists

0 and small motorized vehicles) in the photos taken from the highway (66 photos), local (33) and urban

1 roads (107). Our hypothesis was that in the photos taken on the urban roads, the overall traffic density

2 (including all other visible road users), as well as the amount of light traffic, should be significantly higher

3 than on highways or local roads.

$4 \quad$ Kruskal-Wallis $\mathrm{H}$ Test indicates that there are significant differences in the median number of 5 instances of other road users (vehicles, pedestrians, cyclists, and small motorized vehicle) between the 6 photos from the highway, local and urban roads (Figure 4), $\chi^{2}(2)=15.483, p<0.001$. There were 7 significantly more other road users in the photos taken during touches on the phone on the urban roads 8 compared to highway or local roads (from weak to medium effect, Table 3). 


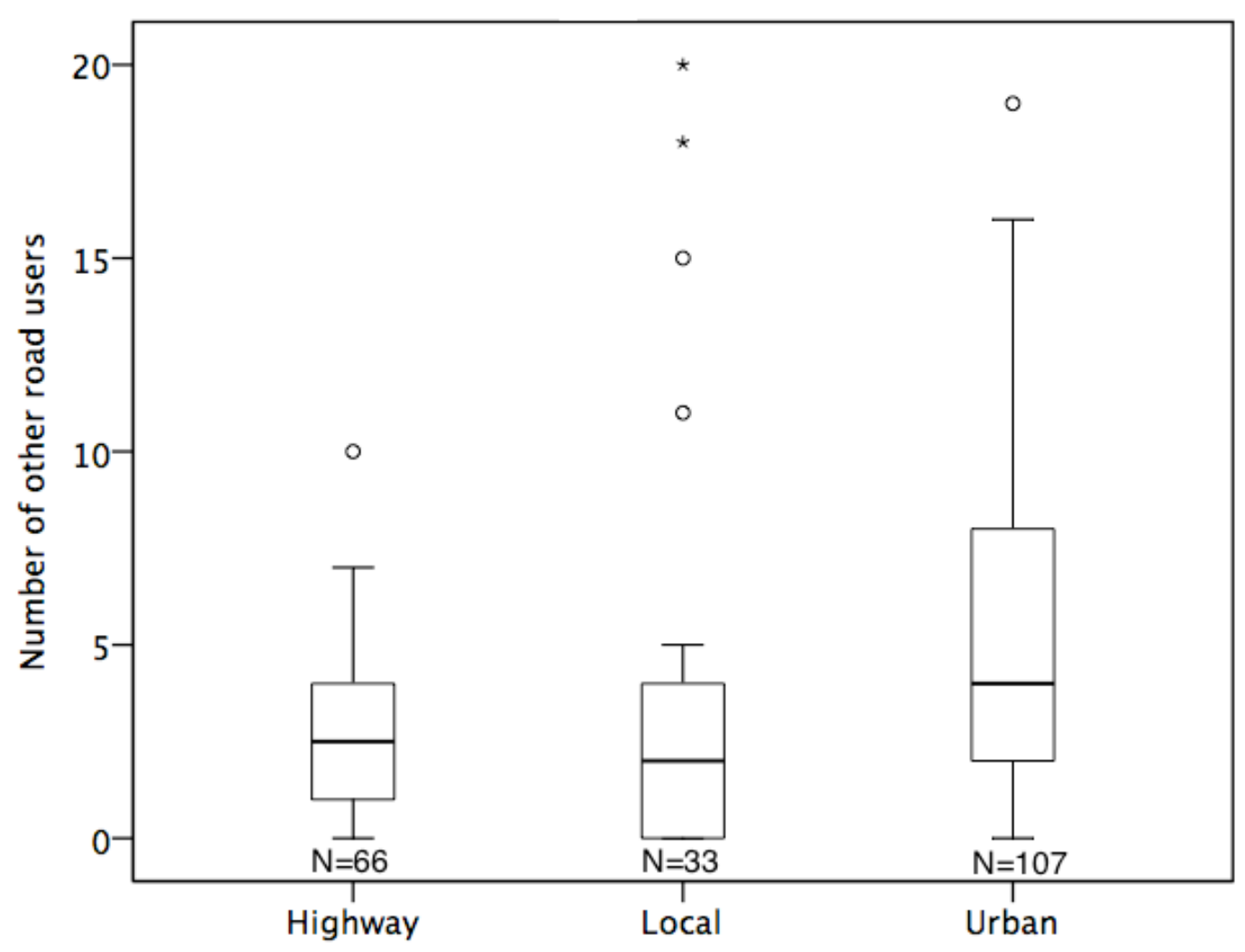

0

2

Fig. 4. Number of other road users in the photos by road type.

Table 3. Results of Kruskal-Wallis $\mathrm{H}$ tests for the median number of other road users in the photos taken during touches on the phone.

Road type $\quad$ Local Urban

Highway

$p>0.99$

$$
p=0.005^{*}(d=0.70)
$$

Local

$$
p=0.004^{*}(d=0.34)
$$

*Significant at $p<0.05$. Cohen's $d$ is calculated for the statistically significant cases.

Respectively, Kruskal-Wallis H Test indicates that there are significant differences in the median

9 number of instances of light traffic (pedestrians, cyclists, and small motorized vehicles) between the

0 photos from the highway, local and urban roads (Figure 5), $\chi^{2}(2)=41.596, p<0.001$. There are significantly

1 more light traffic in the photos taken during touches on the phone on the urban roads compared to highway

2 or local roads (medium-sized effect, Table 4). The significant difference in the median number of 
3 instances of other road users (in total) between the road types can be explained by this difference in light 4 traffic (vehicles only: $p=.104$ ). As expected, there was no light traffic in the photos taken from the 5 highway environments.

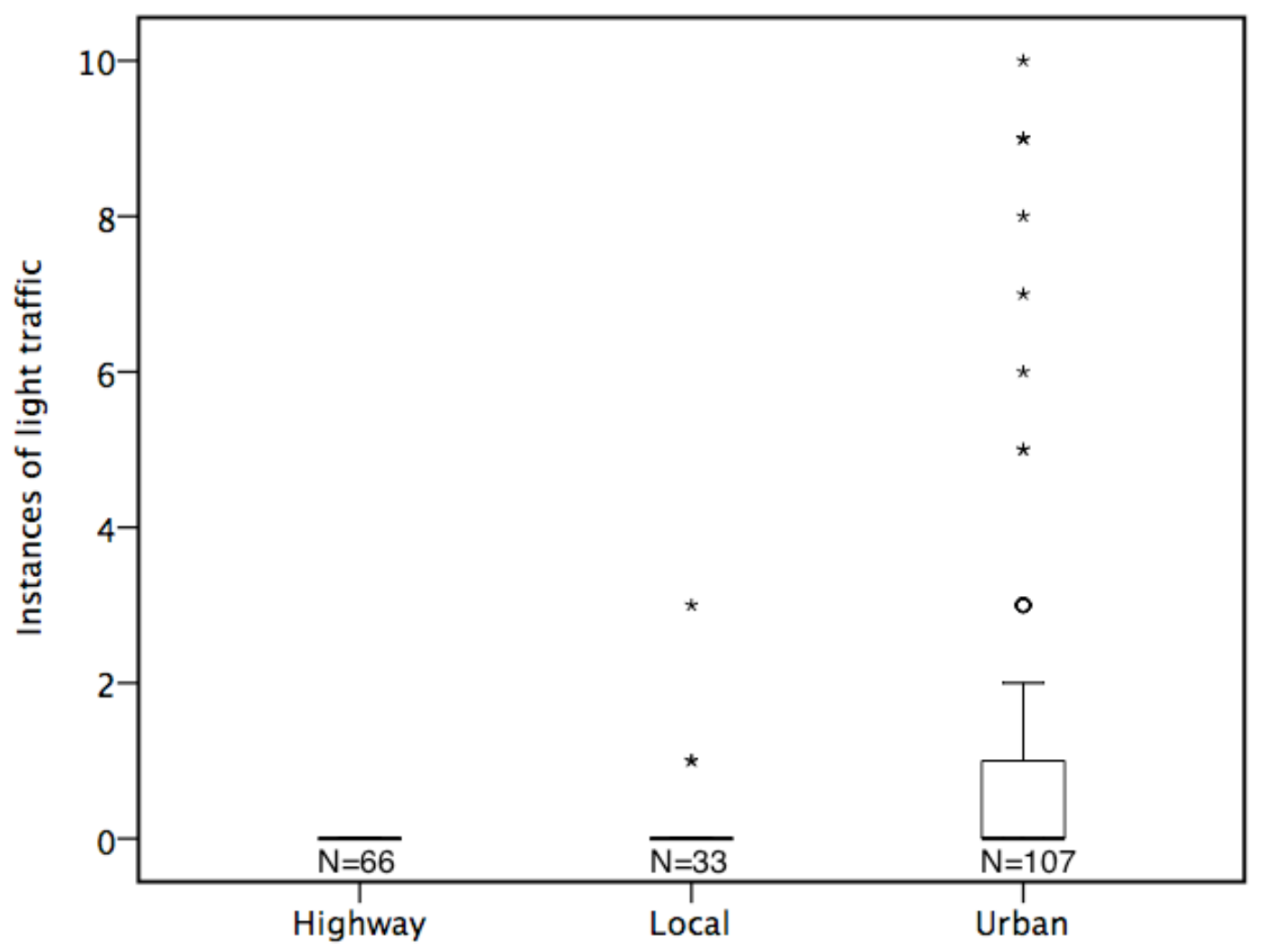

6 8 9 0 1

Fig. 5. Light traffic (pedestrians, cyclists, small motorized vehicles) in the photos by road type.

Table 4. Results of Kruskal-Wallis $\mathrm{H}$ tests for the median number of light traffic in the photos taken during touches on the phone.

\begin{tabular}{|c|c|c|}
\hline Road type & Local & Urban \\
\hline Highway & $p>0.99$ & $p<0.001 *(d=0.76)$ \\
\hline Local & & $p<0.001 *(d=0.65)$ \\
\hline
\end{tabular}

*Significant at $p<0.05$. Cohen's $d$ is calculated for the statistically significant cases. 


\section{$4 \quad 3.4 \quad$ Touches per Hour by Road Type}

5 Statistics for a given road type were compiled only when there were at least 50 touches by a driver for a

6 given road type. The number of touches in in each road can be normalized to the amount of time spent on

7 the given road type. Whenever the car is in motion, the location is updated once a second. Touches per

8 hour can thus be estimated by dividing the number of touches by the number of location fixes and 9 multiplying by 3600 ..

0 As implied by Figure 6, the touches per hour are very strongly non-Gaussian. For the highway data,

1 the Shapiro-Wilks test gives $W=0.62$, which implies non-normality with $p<0.01$. The Wilcoxon rank sum

2 test shows no statistically significant differences between the medians for the various road types; the 3 highway versus local has $p=0.21$, while the rest are much higher (Table 5).

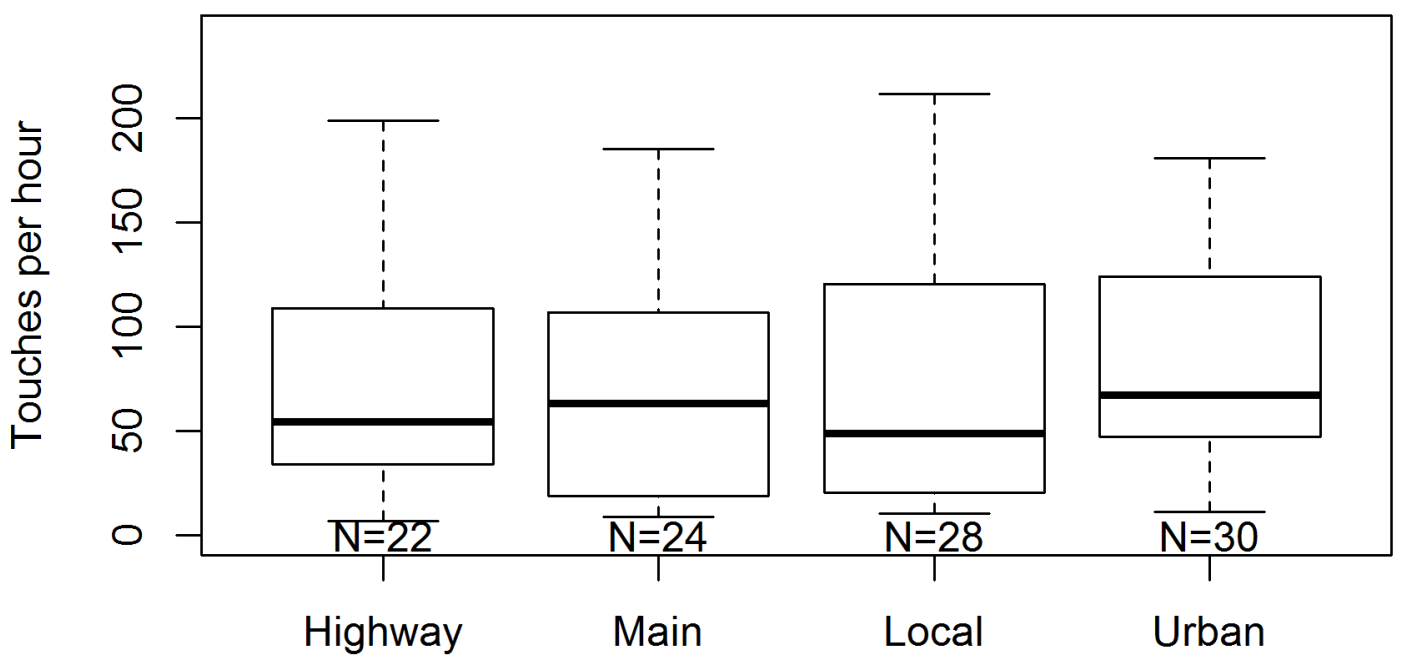

5 Fig. 6. Touches per hour by the four different road types (data was included only if the driver had at least 6 50 touches on the given road type). 
Table 5 Touches per hour on the different road types

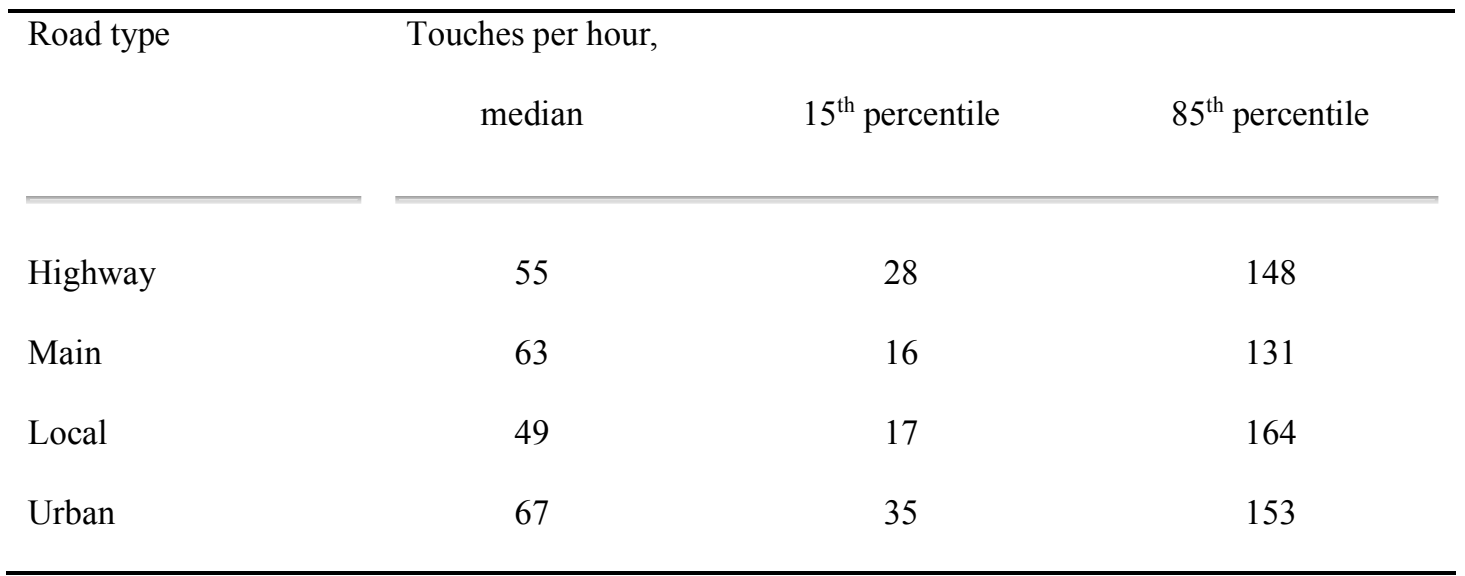

2 Within-subject variations were controlled by dividing each driver's touch density (i.e. touches per 3 hour) by the driver's mean touch density over the whole experiment (Figure 7, Table 6). Wilcoxon rank 4 sum tests (Table 7) show that the normalized median of the urban roads is larger than the other road types, 5 the effect sizes being large. Thus, drivers tended to touch their phones more while driving on urban roads.

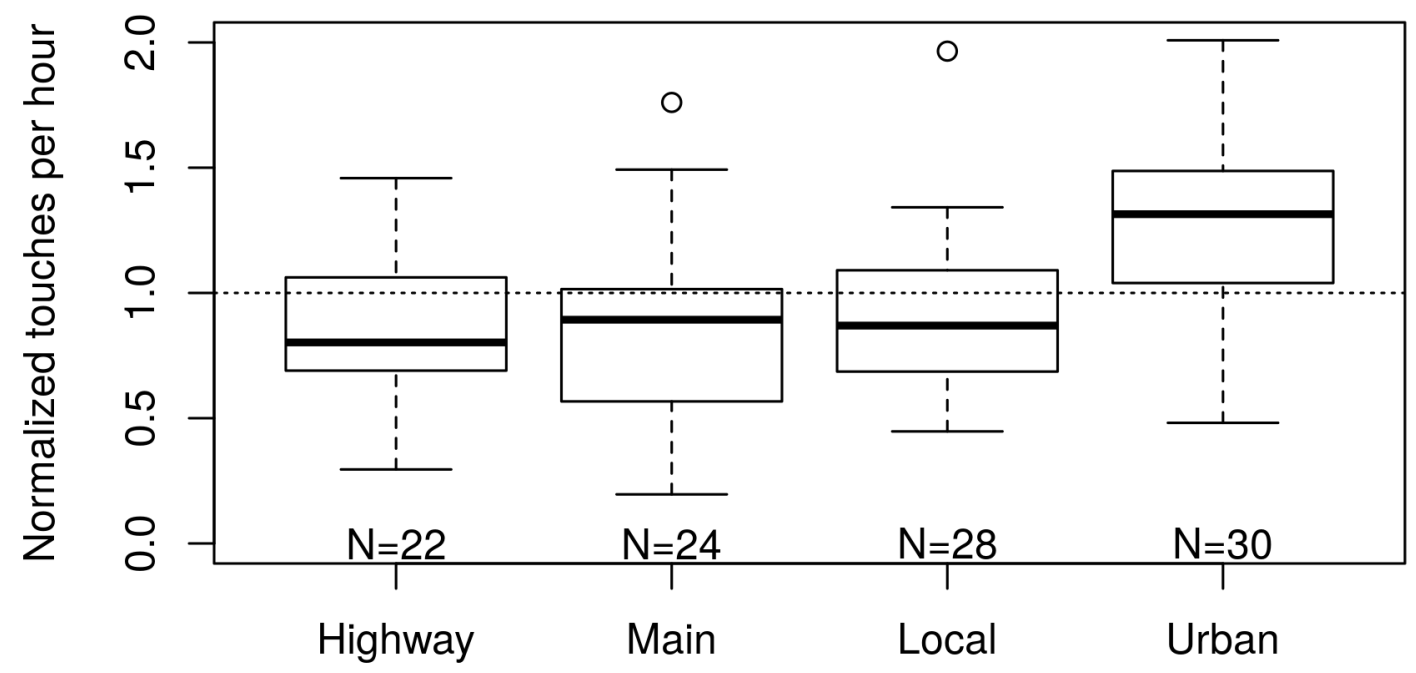

Fig. 7. Normalized touches per hour by road type. 
9 Table 6 Normalized touches per hour on the different road types

0

\begin{tabular}{lccc}
\hline Road type & $\begin{array}{c}\text { Norm touches per hour, } \\
\text { median }\end{array}$ & $15^{\text {th }}$ percentile & $85^{\text {th }}$ percentile \\
\cline { 1 - 2 } & 0.80 & 0.58 & 1.25 \\
Highway & 0.89 & 0.54 & 1.16 \\
Main & 0.87 & 0.67 & 1,17 \\
Local & 1.31 & 0.86 & 1.692 \\
Urban & & & \\
\hline
\end{tabular}

2

3 Table 7. Results of Wilcoxon rank sum tests for normalized touches per hour by road type 4

\begin{tabular}{llll}
\hline Road type & Main & Local & Urban
\end{tabular}

\begin{tabular}{|c|c|c|c|}
\hline Highway & $p=0.62$ & $p=0.78$ & $p<0.001 *(d=1.05)$ \\
\hline Main & & $p=0.25$ & $p=<0.001 *(d=1.08)$ \\
\hline Local & & & $p<0.001 *(d=0.94)$ \\
\hline
\end{tabular}

*Significant at $p<0.05$. Cohen's $d$ is calculated for the statistically significant cases.

6

$7 \quad 3.5 \quad$ Analysis of Application Use Instances

8 We defined application use instances by clustering the touches. If two touches were separated by less than

930 seconds, they were considered to be part of the same cluster. The 30 -second threshold is based on the

0 on-road data of Blanco et al. [1], in which the longest and most complicated in-car tasks lasted for 30

1 seconds on average, and the naturalistic driving data of Christoph and van Nes [3], in which the average

2 duration of manual interactions with a mobile phone was 31.0 seconds. The most frequently occurring

3 FrontApp tag in the cluster is considered to be the main application used in the cluster. Especially on

4 urban roads, drivers may perform part of the task while stationary or braking to stationary. In this analysis,

5 we have included only instances, which start when the car is moving with a speed of at least $2 \mathrm{~m} / \mathrm{s}$ during 
6 at least one touch. The data are normalized to uses per hour by the same method that was used to derive

7 touches per hour (Figure 8).

8 The data are again non-Gaussian, with a Shapiro-Wilkes test for the highway data giving $W=0.92$,

9 implying non-normality with $p=0.038$. Non-parametric tests thus need to be performed again. The median

0 is 4.1 and 15-85 percentile limits are 2.2 and 9.5. A typical driver in our data set thus uses some

1 application every fifteen minutes or so, while the heaviest users are using their phones almost every five

2 minutes. There were clear differences between road types (Figure 9, Tables 8 and 9), application usage per

3 hour being significantly more frequent on urban roads compared to the other road types (medium to large 4 effect).

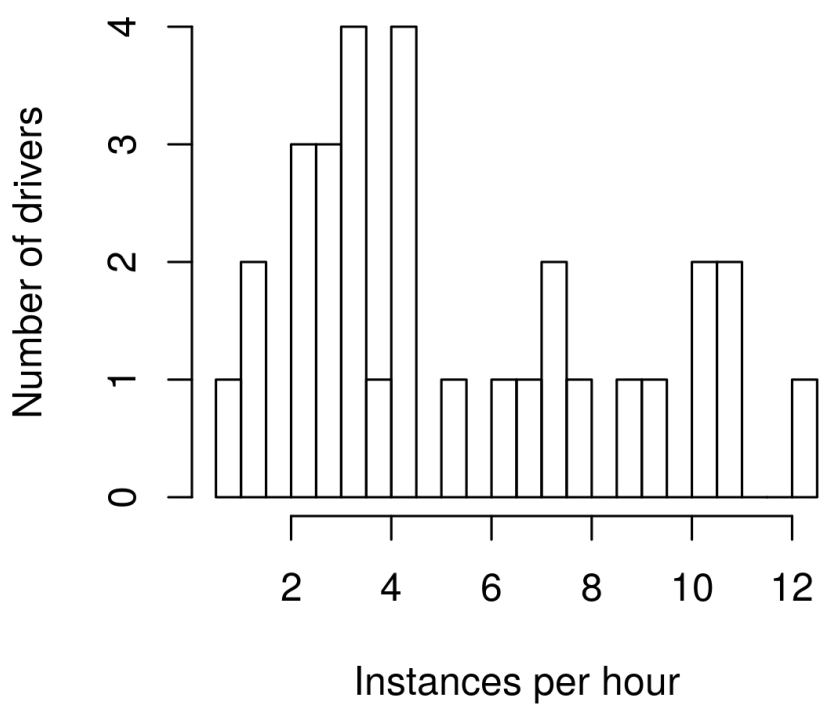

Fig. 8. Application use instances per hour, $N=30$. 


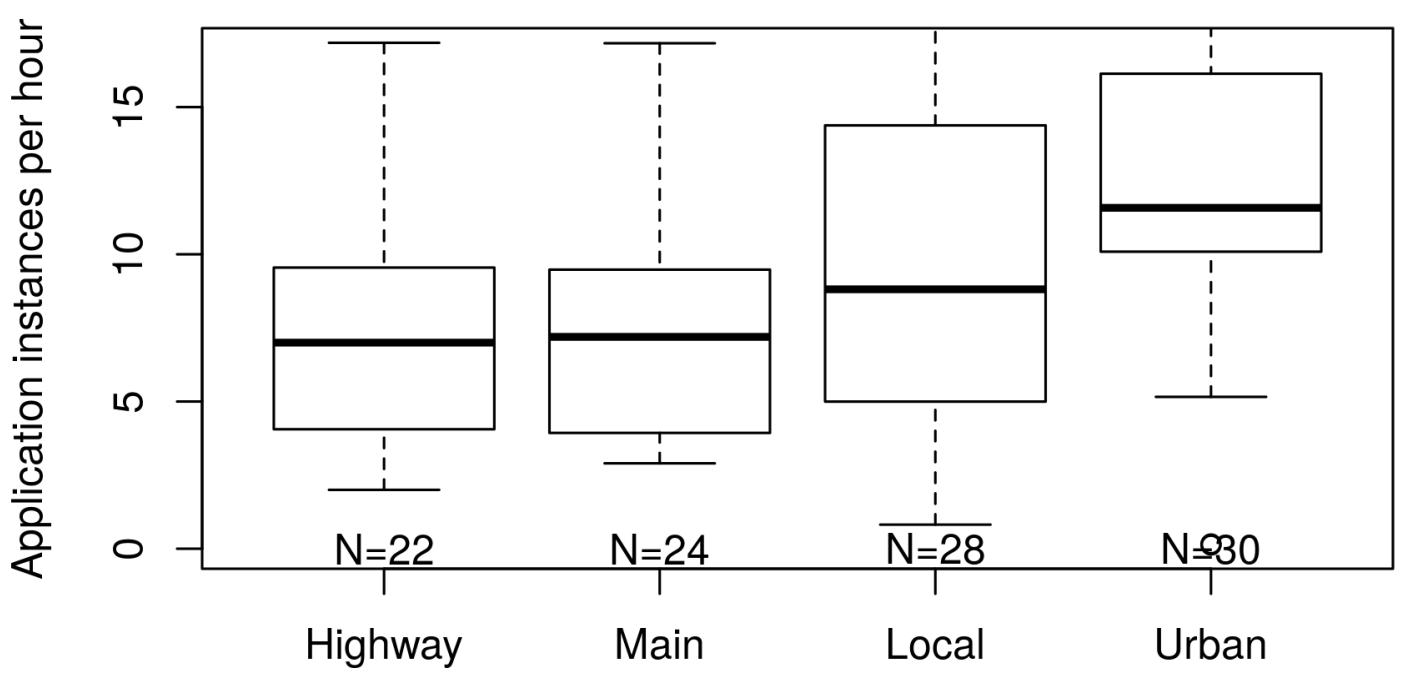

Fig. 9. Application use instances per hour by road type.

0

1 Table 8 Application use instances per hour on the different road types

\begin{tabular}{lccc}
\hline Road type & $\begin{array}{c}\text { Application use } \\
\text { instances per hour, } \\
\text { median }\end{array}$ & $15^{\text {th }}$ percentile & $85^{\text {th }}$ percentile \\
\cline { 3 - 4 } & & & \\
\cline { 2 - 4 } & 7.0 & 3.4 & 13.7 \\
Highway & 7.2 & 3.8 & 13.5 \\
Lain & 8.8 & 4.4 & 16.2 \\
Urban & 11.5 & 8.4 & 19.0 \\
\hline
\end{tabular}


Table 9 Results of Wilcoxon rank tests for application use instances per hour by road type

\begin{tabular}{|c|c|c|c|}
\hline Road type & Main & Local & Urban \\
\hline Highway & $p=0.99$ & $p=0.21$ & $p<0.001 *(d=0.90)$ \\
\hline Main & & $p=0.19$ & $p<0.001 *(d=0.93)$ \\
\hline Local & & & $p=0.045^{*}(\mathrm{~d}=0.48)$ \\
\hline
\end{tabular}

*Significant at $p<0.05$. Cohen's $d$ is calculated for the statistically significant cases.

The duration of each application use instance can also be estimated as the time between the first and

8 the last touches in a cluster. There are statistically significant differences between the road types (Figure

$9 \quad 10$ and Table 10). According to the Shapiro-Wilks test, the data are too skewed to make an ANOVA

0 comparison. However, a Wilcoxon rank test (Table 11) shows that task durations are significantly longer

1 on highways, main, or local roads than on urban roads. The effect size is medium to strong (Cohen's d up

2 to 0.84 ). Application use instance durations tended to be shorter on urban roads than on the other road 3 types.

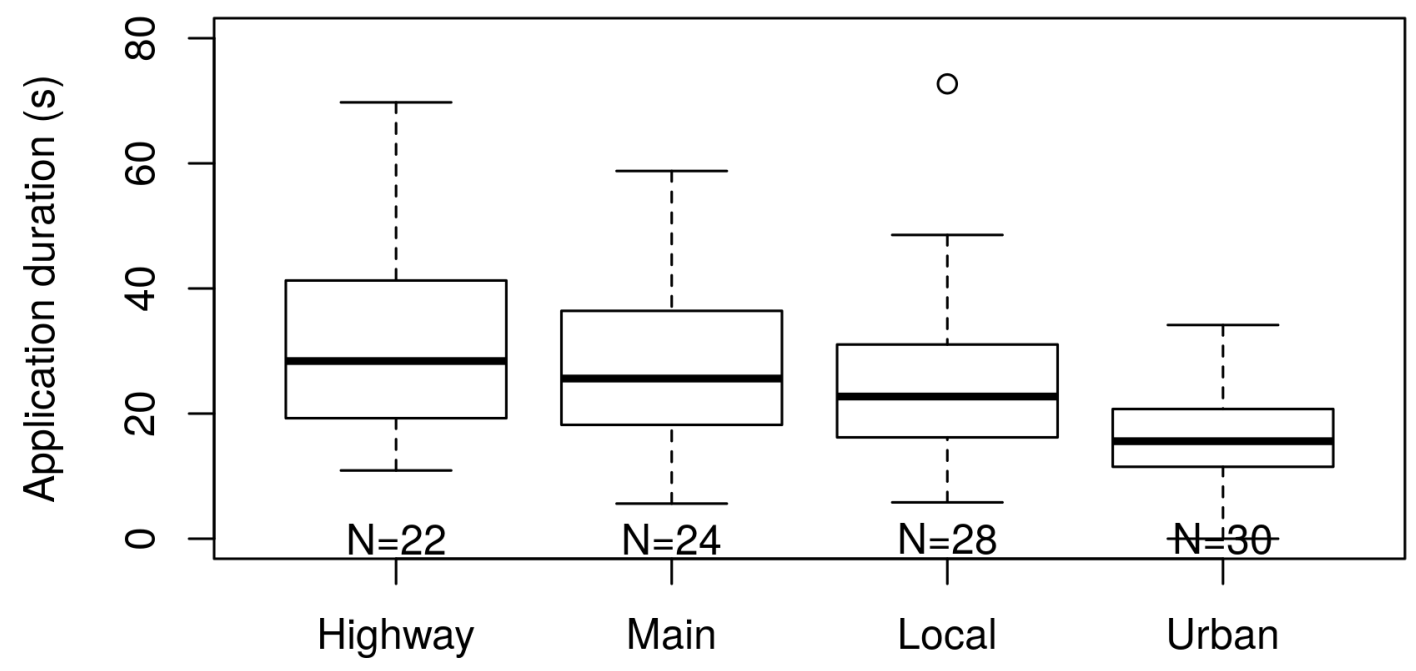

Fig. 10. Duration of application use instances by road type (s). 
Table 10 Duration of application use instances on the different road types

\begin{tabular}{lccc}
\hline Road type & Median duration (s) & $15^{\text {th }}$ percentile & $85^{\text {th }}$ percentile \\
\cline { 1 - 3 } & 28.4 & 16.7 & 56.2 \\
Highway & 25.7 & 13.9 & 42.8 \\
Main & 22.7 & 15.4 & 34.1 \\
Local & 15.6 & 10.0 & 23.6 \\
Urban & & \\
\hline
\end{tabular}

Table 11. Results of Wilcoxon rank tests for mean duration of application use instances by road type

\begin{tabular}{llll}
\hline Road type & Main & Local & Urban
\end{tabular}

\begin{tabular}{|c|c|c|c|}
\hline Highway & $p=0.39$ & $p=0.17$ & $p<0.001 *(d=0.84)$ \\
\hline Main & & $p=0.59$ & $p=0.002 *(d=0.68)$ \\
\hline Local & & & $p=0.002 *(\mathrm{~d}=0.46)$ \\
\hline
\end{tabular}

*Significant at $p<0.05$. Cohen's $d$ is calculated for the statistically significant cases.

\section{$3 \quad 3.6 \quad$ Frequently Used Applications}

4 The application analysis is complicated somewhat by the fact that on different Android phone models, the

5 FrontApp listed may be different for the same application, or in some cases the same for different

6 applications. For example, Spotify use can be marked by at least three different FrontApp values.

The actual number of application use instances per application per user is also relatively small. Thus,

8 it is not possible to make reliable comparisons between different drivers or road types. However, accurate

9 aggregate statistics can be collected, that is, combined statistics from all users over the entire control 0 period (see Table 12).

1 The most used applications were also used at all speeds. PokémonGo was an exception; it was

2 played mostly at lower speeds, as could be expected from the game mechanics. The median speed during 3 an instance of PokémonGo usage is just $21 \mathrm{kmh}$; that is, it was very often played at crawling speeds.

4 However, there are also some instances of use at higher speeds (19 above $60 \mathrm{kmh}$ ). By contrast, for 
5 instance, WhatsApp was used almost evenly at all speeds (median $57 \mathrm{kmh}$ ). All of the frequently used 6 downloadable applications are also among the 100 most downloaded applications in Google Play in 7 Finland, which suggests the applications drivers use in the car are the same applications they use in 8 general. However, naturally the frequency of use for driving-related applications, such as navigation 9 applications, could be higher than outside the car. 
Table 12 Statistics for the most frequently used applications

\begin{tabular}{|c|c|c|c|c|c|c|}
\hline Application & $N$ instances & $\begin{array}{c}\text { Touches/instance } \\
\text { median }(15 \%-85 \%)\end{array}$ & $\begin{array}{c}\text { Duration (s) } \\
\text { median }(15 \% \text { - } \\
85 \%)\end{array}$ & $\begin{array}{c}\text { Speed kmh } \\
\text { median (15\%- } \\
85 \%)\end{array}$ & $\begin{array}{l}N \text { drivers } \\
\text { using }\end{array}$ & $\begin{array}{c}\text { Time } \\
\text { distracted } \\
\text { (h) }\end{array}$ \\
\hline Contacts & 818 & $3(1-15)$ & $17(4-57)$ & $56(20-94)$ & 27 & 7.5 \\
\hline Whatsapp & 622 & $8(1-76$ & $35(9-112)$ & 57 (16-95) & 23 & 10.1 \\
\hline Music & 366 & $4(1-17-)$ & $17(3-48)$ & $68(25-92)$ & 12 & 2.9 \\
\hline Maps & 277 & $3(1-13)$ & $11(1-42)$ & $60(18-96)$ & 19 & 2.2 \\
\hline Facebook & 215 & $7(2-39)$ & $31(10-96)$ & 68 (21-99) & 15 & 3.5 \\
\hline PokémonGo & 175 & $26(5-152)$ & $141(30-637)$ & $21(10-48)$ & 4 & 13.9 \\
\hline Search & 169 & $9(3-37)$ & $34(12-83)$ & $52(10-94)$ & 11 & 2.5 \\
\hline Dialer & 118 & $3(1-15)$ & $19(4-65)$ & $53(15-99)$ & 12 & 1.0 \\
\hline Browser & 104 & $8(2-29)$ & $37(5-93)$ & $67(27-94)$ & 15 & 1.6 \\
\hline MMS & 92 & $5(1-36)$ & $28(6-97)$ & $49(18-92)$ & 12 & 1.5 \\
\hline YouTube & 63 & $8(1-32)$ & $24(1-78)$ & $82(29-89)$ & 3 & 0.7 \\
\hline Email & 59 & $8(2-35)$ & $45(14-104)$ & $74(24-109)$ & 14 & 1.2 \\
\hline Banking & 36 & $6(2-19)$ & $28(6-64)$ & $56(19-93)$ & 12 & 0.5 \\
\hline Email & 59 & $8(2-35)$ & $45(13-130)$ & $74(24-109)$ & 14 & 1.2 \\
\hline Netflix & 33 & $3(1-10)$ & $10(1-41)$ & $83(59-86)$ & 3 & 0.2 \\
\hline Calendar & 32 & $8(2-14)$ & $37(18-134)$ & $61(8-84)$ & 12 & 0.6 \\
\hline Instagram & 24 & $8(2-72)$ & $34(7-128)$ & $73(31-100)$ & 6 & 0.4 \\
\hline Camera & 20 & $5(1-34)$ & $33(3-65)$ & $50(18-91)$ & 12 & 0.3 \\
\hline Snapchat & 19 & $8(3-33)$ & $28(7-69)$ & $41(20-59)$ & 2 & 0.2 \\
\hline Gallery & 18 & $4(2-39)$ & $31(6-148)$ & $40(14-68)$ & 6 & 0.3 \\
\hline Fonecta* & 20 & $92-22)$ & $33(9-101)$ & $54(15-83)$ & 6 & 0.3 \\
\hline News & 9 & $5(2-10)$ & $16(1-40)$ & $57(28121)$ & 3 & 0.1 \\
\hline Outlook & 8 & $5(3-22)$ & $35(17-102)$ & $86(64-98)$ & 2 & 0.2 \\
\hline Twitter & 7 & $15(6-76)$ & $75(25-320)$ & $79(33-98)$ & 2 & 0.4 \\
\hline Tinder & 4 & $12(5-26)$ & $32(25-78)$ & 45 (41-49) & 2 & 0.1 \\
\hline
\end{tabular}


4 The ratio of time spent distracted can - in principle - be estimated by adding the durations of the 5 application use instances and dividing by the time spent driving (Tables 12 and 13, see Figure 11 for 6 percentages per road type). Note that this estimate may be somewhat distorted, since the task duration may 7 include some cases in which the car has been stopped during part of the task execution. In any case, 8 Shapiro-Wilks tests show no significant differences between the road types in the ratio of time spent 9 distracted.

0 Table 13 Approximate ratio of time spent distracted on the different road types 1

\begin{tabular}{|c|c|c|c|}
\hline Road type & Ratio median & $15^{\text {th }}$ percentile & $85^{\text {th }}$ percentile \\
\hline Highway & 0.063 & 0.027 & 0.17 \\
\hline Main & 0.052 & 0.018 & 0.11 \\
\hline Local & 0.053 & 0.018 & 0.15 \\
\hline Urban & 0.062 & 0.026 & 0.091 \\
\hline
\end{tabular}

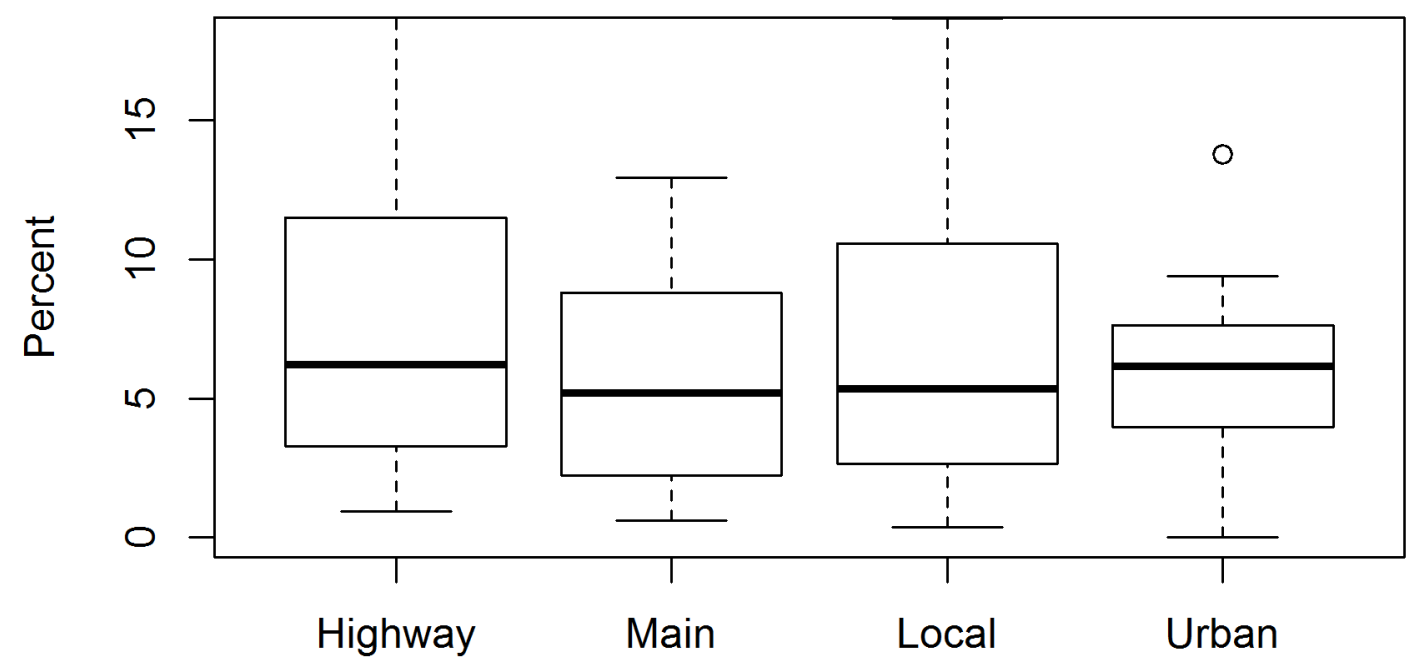

Fig. 11. Percentage of time spent distracted by application use by road type, $N=30$. 
The "Time distracted (h)" column in Table 12 can be used to make a very rough estimate of the

6 distraction level caused by the application in question. It is the sum of all the durations of all the 7 application use instances during the study. The total amount of time spent driving by all the drivers during 8 the control period was 1724 hours. If the driving was done by a single driver, the driver would have been 9 distracted by the Contacts application for 7.5 hours during this time, by WhatsApp for 10.1 hours, and so 0 on.

Two applications dominate the time distracted almost completely: Whatsapp and PokémonGo, both 2 causing over 10 hours of distracted driving (out of 1724 hours). Note that Facebook Messenger is known 3 to be missing from this list, as are the traditional SMS text messages as well.

$4 \quad$ These values are primarily useful as a measure of the relative distraction caused in the overall 5 population by any given application. The exact hours given may be an overestimate, as we count an 6 application as occurring in motion when at least one touch has a registered speed of more than $2 \mathrm{~m} / \mathrm{s}$; thus, 7 some cases may be included where the driver was already cruising to a stop after started touching the 8 phone. The histogram below (Figure 12) shows that this does indeed affect the PokémonGo results, as 9 could be expected from the game mechanics. The median speed during an instance of PokémonGo usage 0 is just $22 \mathrm{kmh}$; that is, it is very often played at crawling speeds. However, there are also instances of use 1 at higher speeds. By contrast, WhatsApp is used almost evenly at all speeds (median 63 kmh, see Figure $213)$. 
PokemonGo instances, mean speed

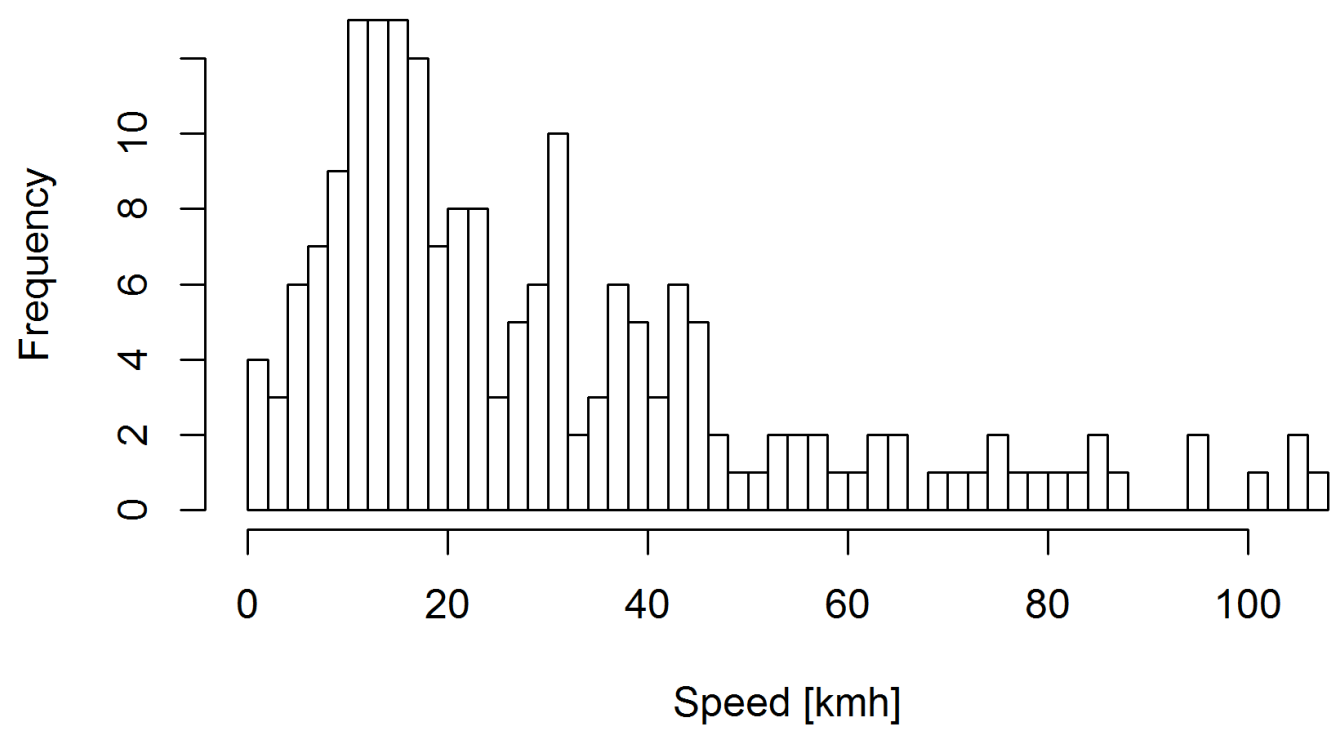

Fig. 12. Speed distribution for use instances of PokémonGo, $N=175$.

WhatsApp instances, mean speed

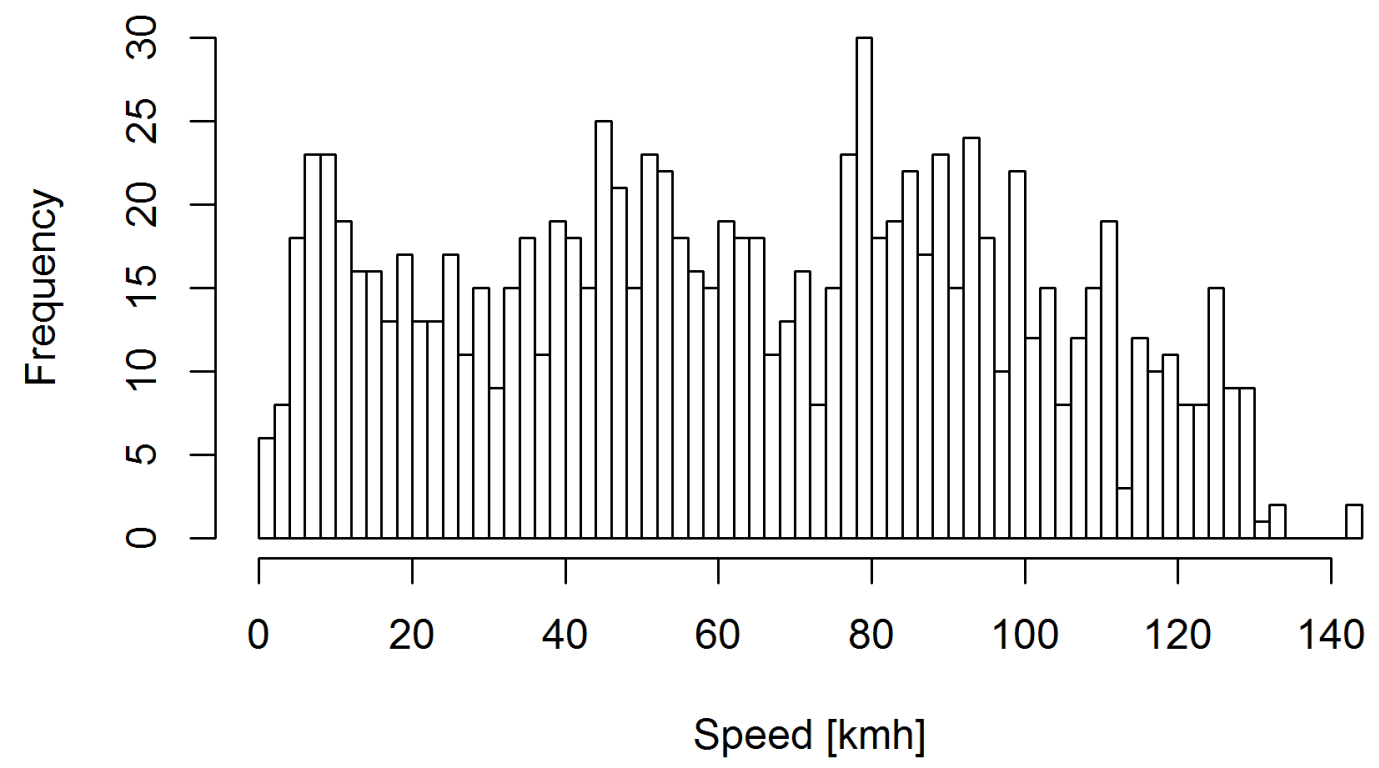

Fig. 13 Speed distribution for use instances of WhatsApp, $N=622$. 
By contrast to WhatsApp, navigation-related applications are associated with clearly smaller time

0 distracted even though these are used frequently. This implies that they may cause a much smaller risk to

1 traffic safety due to their distraction potential. The user interfaces of navigation applications may to some

2 extent been optimized for use while driving, and interactions tend to be short. By contrast, for instance,

3 WhatsApp is designed to be used when stationary, and has no features that would allow it to be optimized

4 for in-car use by the driver.

\section{4. Discussion}

6 Our main research question was whether we could observe any statistically significant differences between

7 smartphone usages on different road types by the heavy-user participants. Our data suggest that drivers

8 modulate their phone use behavior depending on road type. Drivers produced significantly more touches to

9 the smartphone per hour on urban roads (large effect size), while the time duration spent on any given

0 application instance was shorter on urban roads (from medium to large effect).

The effect on the touches per hour is in fact in the opposite direction to our hypothesis. Urban roads

2 present the most visually demanding driving scenario, and we would have expected to see a decrease in

3 phone usage in these road environments. The driving speeds are significantly lower in urban roads than on

4 highway or main roads but the density of crossing traffic in the form of cars, cyclists and pedestrians is

5 significantly higher on the urban roads (at least in Finland). The analysis of traffic densities in the photos

6 taken by each touch on the drivers' phones indicates that the drivers also used their phones in such traffic

7 situations, in which the traffic density between the road types varied in accordance with this expectation.

8 There was significantly more light traffic (pedestrians, cyclists and small motorized vehicles) in front of

9 the vehicle with access to the road the driver was using while interacting with the smartphone on the urban

0 roads compared to the highway or local roads. If the probability of unexpected events is assumed to

1 correlate with traffic density and the amount of light traffic on the road environment, then one could argue

2 the urban roads place the highest attentional demand for the driver (of the studied road environments). As 
3 indicated in [8], highway road environment with no crossing traffic (or light traffic) should present the

4 least visual demand, and thus we expected to see more touches and application use on highways and main

5 roads than on urban roads. A similar opposite finding was made by Huth et al. [6] in a smaller naturalistic

6 study $(N=9)$ in France, suggesting that the drivers used their phones more in urban road environments than

7 on highway or rural roads.

8 The experiment by Horrey and Lesch [5] indicated that although their drivers seemed to be aware of

9 the demands of the driving situation ahead, the drivers did not tend to postpone the presented secondary

0 tasks even if they were given the chance. One explanation for these types of behaviors, similar to our

1 findings, could be the impairment of situation awareness due to the cognitive demands of the secondary in-

2 car tasks [18].

3 Alternative explanation could relate to the tendency to use the smartphone while stopped (or 4 stopping) for traffic lights or while driving in congestion. Our definition of "application use instance" 5 becomes problematic in urban traffic, where the stops may be frequent. The more frequent stops on the 6 urban roads may encourage the use of the smartphone. At a general level, there were as many touches on 7 the smartphone made while driving as while stationary. The shorter instances of use in urban traffic 8 compared to the other road types could suggest some of the usage were allocated together with brief stops.

9 The negative safety or other types of distraction effects (e.g. delayed reaction times to changing traffic 0 lights) related to this kind of behavior are unknown. It may also be simply that due to the more rapidly 1 changing situations in the urban traffic, the drivers have to interrupt their phone usage more frequently 2 than, for instance, on less congested highways. This would suggest they do not actively moderate their 3 phone usage based on the changing demands of the traffic situation but passively react to these. The 4 alternative explanations for the observed behaviors should be studied more carefully in future research.

5 Contacts and WhatsApp were the most commonly used individual applications. The touch and 6 duration data for those two applications were, however, quite different. A single instance of WhatsApp 
7 interaction included a median of 8 touches and lasted for 35 seconds; a Contacts interaction included a

8 median of 3 touches and lasted for 17 seconds. We can assume that the number of touches and duration of

9 a use instance are related to the risk caused by the distraction.

0 This suggests that not all applications are equally risky. A good example of a relatively safe 1 application is Maps, where a typical interaction required just 3 touches and 11 seconds. The design of 2 navigation applications (at least implicitly) takes into account the fact that drivers use them while driving, 3 and hence visual-manual interactions are often minimized. However, one should realize the apparent 4 usability paradox here; making an application more easily (and safely) usable while driving may 5 encourage and increase the frequency of use, which may again increase the overall risk of distraction.

Possibly our most surprising finding was that four drivers used PokémonGo so heavily that the 7 application rose to be sixth on the most frequently used applications list by the total number of touches.

8 Furthermore, the interactions during this application were particularly long (median 141 seconds with 26 9 touches). The result implies that high distraction and thus, risk levels, are accepted by some of our heavy 0 users. The application was mainly used at low speeds but this may be due more to the game mechanics 1 (targeting) instead of risk-related adaptive behaviors.

All our participants were drivers who drive a lot and admit to use their smartphones frequently while 3 driving. The findings may not represent the behaviours of drivers who drive less frequently or use their 4 smartphones while driving less frequently. In addition, the drivers were Finnish and there may be cultural 5 differences in multitasking behaviours behind the wheel [19]. The Finnish road system is fairly simple and 6 there are much less road users per kilometre, in particular on highways, than, for instance, in the United 7 States or in China. There is a need for similar studies in different traffic cultures.

For future studies, location data could be examined in greater detail for finding possible patterns on 9 which kind of locations people tend to use and modulate their phones while driving (if they do). 
0 Furthermore, acceleration data could be examined for the relationships between touches and vehicle speed

1 state as well as lateral movements and possible safety-critical events [7].

Another limitation of our study was the absence of eye-tracking. We were not able to analyse if the

3 drivers modulated their in-car glance durations based on the demands of the traffic scenario, as suggested

4 by [12], [16] and [17]. For the phone use instances and the number of touches the evidence is, however,

5 clear; our drivers did not adapt their smartphone usage according to the attentional demands of the road

6 environment but even an opposite effect was found. This finding suggests that context-sensitive distraction

7 warnings (e.g., [9]), which were studied in the larger experiment, could be useful, if effective, in making

8 drivers to better adapt their phone usage according to the demands of the driving situation.

\section{5. Conclusions}

0 The naturalistic phone usage of 30 car drivers was monitored in between June and September 2016 in

1 Finland. All of the drivers were volunteers who admitted to using their smartphones frequently while

2 driving. However, there were large variations in the smartphone usage between the drivers. Touches per

3 hour could be unambiguously determined; the median value was 41 touches per hour (15th percentile 13 ,

4 85th percentile 142). The number of application instances per hour could be estimated from the touches;

5 the median value was 4.1 (15th percentile $2.2,85$ th percentile 9.5$)$. Since the drivers were not selected

6 from a random sample, these figures cannot be generalized to the general population but represent the

7 Finnish smartphone heavy-users in traffic.

The data showed an unexpected tendency in phone usage: drivers tended to make more touches per

9 hour on urban roads than on other road types. However, a statistically significant difference was seen see

0 in the time duration spent on individual applications: on highways the median duration of an application

1 use instance was 28.4 seconds, while on urban roads the median was 15.6 seconds.

The applications used by the drivers could be identified, though with some caveats. The two single

3 most used application, by a rather large margin, were Contacts and the WhatsApp messaging application. 
4 One instance of WhatsApp also required a large number of touches (median 8). By contrast, Contacts 5 required and another commonly used application, Maps, required only a median of 3 touches per use

6 instance. This suggests that not all applications are equally risky in terms of distraction. A surprising find

7 was that PokémonGo was one of the most heavily used applications in traffic as measured by the total 8 number of touches (a small minority of drivers using it very heavily).

9 The findings indicate, unexpectedly, that Finnish drivers who use their smartphones while on the

0 move use their phones more frequently while driving in urban conditions. The usage pattern may however

1 be slightly different, with application uses being performed more rapidly in the urban conditions.

\section{Acknowledgments}

3 We thank Ficonic Solutions Ltd of Jyväskylä, Finland, for designing the software used in this experiment 4 and Paula Paananen for the help with the analysis of the photo material. This work was supported by the 5 VisGuard project, funded by TEKES (Finnish Funding Agency for Technology and Innovation; Grant 6 TEKES Dnro 813/31/2015) and the University of Jyväskylä.

\section{$7 \quad$ References}

[1] Blanco, M., Biever, W. J., Gallagher, J. P., \& Dingus, T. A. (2006). The impact of secondary task cognitive processing demand on driving performance. Accident Analysis \& Prevention, 38, 895-906.

[2] Burnett, G.E. \& Porter, J.M. (2001). Ubiquitous computing within cars: designing controls for non-visual use. International Journal of Human Computer Studies, 55, 521-531.

[3] Christoph, M. \& van Nes, N. (2013). When do drivers use their mobile phone? An analysis on the context of mobile phone use based on naturalistic driving data. In Proceedings of the 3rd International Conference on Driver Distraction and Inattention (oral presentation 81-O).

[4] Hancox, G., Richardson, J., \& Morris, A. (2013). Drivers' willingness to engage with their mobile phone: The influence of phone function and road demand. IET Intelligent Transport Systems, 7(2), 215-222.

[5] Horrey, W.J., \& Lesch, M.F. (2009). Driver-initiated distractions: Examining strategic adaptation for in-vehicle task initiation. Acc. Anal. Prev. 41, 115-122.

[6] Huth, V., Tattegrain, H., Sanchez, Y., \& Brusque, C. (2013). Deciding to be distracted: Drivers' strategic choices to interact with the mobile phone. In Proceedings of the 3rd International Conference on Driver Distraction and Inattention (oral presentation 41-O). 
[7] Klauer, S.G., Dingus, T.A., Neale, V.L., et al. (2006). The Impact of Driver Inattention On Near-Crash/Crash Risk: An Analysis Using the 100-Car Naturalistic Driving Study Data. Washington, DC: National Highway Traffic Safety Administration.

[8] Kujala, T., Mäkelä, J., Kotilainen, I., \& Tokkonen, T. (2016). The attentional demand of automobile driving revisited - Occlusion distance as a function of task-relevant event density in realistic driving scenarios. Human Factors, 58, 163-180.

[9] Kujala, T., Karvonen, H., \& Mäkelä, J. (2016). Context-sensitive distraction warnings - Effects on drivers' visual behavior and acceptance. International Journal of Human-Computer Studies, 90, 39-52.

[10] Kujala, T. \& Salvucci, D.D. (2015). Modeling visual search on in-car displays: The challenge of modeling safety-critical lapses of control. International Journal of Human-Computer Studies, 79, 66-78.

[11] Lee, J.D., Roberts, S.C., Hoffman, J.D., \& Angell, L.S. (2012). Scrolling and driving: how an MP3 player and its aftermarket controller affect driving performance and visual behavior. Human Factors 54, 250-263.

[12] Metz, B., Schömig, N., \& Krüger, H. P. (2011). Attention during visual secondary tasks in driving: Adaptation to the demands of the driving task. Transportation Research Part F: Traffic Psychology and Behaviour, 14(5), 369380 .

[13] Metz, B., Landau, A., Hargutt, V., \& Neukum, A. (2013). Exposure to secondary tasks in germany: Results from naturalistic driving data. In Proceedings of the 3rd International Conference on Driver Distraction and Inattention (oral presentation 35-O).

[14] Salvucci, D.D. \& Kujala, T. (2016). Balancing structural and temporal constraints in multitasking contexts. In Proceedings of the 38th Annual Meeting of the Cognitive Science Society (CogSci 2016), 2465-2470.

[15] Victor, T., Dozza, M., Bärgman, J., et al. (2015). Analysis of Naturalistic Driving Study Data: Safer Glances, Driver Inattention, and Crash Risk (No.S2-S08A-RW-1). Washington, DC: Transportation Research Board.

[16] Wierwille, W.W. (1993). An initial model of visual sampling of in-car displays and controls. In: Gale, A.G., Brown, I.D., Haslegrave, C.M., Kruysse, H.W., Taylor, S.P. (Eds.), Vision in Vehicles IV (pp. 271-279).

Amsterdam, NL: Elsevier Science.

[17] Wikman, A. S., Nieminen, T., \& Summala, H. (1998). Driving experience and time-sharing during in-car tasks on roads of different width. Ergonomics, 41(3), 358-372.

[18] Young, K.L. \& Salmon, P.M. (2012). Examining the relationship between driver distraction and driving errors: a discussion of theory, studies and methods. Saf. Sci. 50, 165-174.

[19] Özkan, T., Lajunen, T., Chliaoutakis, et al. (2006). Cross-cultural differences in driving behaviours: A comparison of six countries. Transportation Research Part F: Traffic Psychology and Behaviour, 9(3), 227-242. 